\title{
VARICELLA ENCEPHALITIS
}

\author{
BY \\ J. D. HEPPleston, K. M. PEARCE and P. O. YATES \\ From Hope Hospital, Salford and the Department of Pathology, University of Manchester
}

(RECEIVED FOR PUBLICATION FEBRUARY 12, 1959)

The encephalitis which may complicate exanthematous fevers usually shows considerable perivascular demyelination of the white matter and for this reason is often thought of as primarily a disorder of the myelin. There is still some doubt as to whether the encephalitis of chickenpox is of this general type since although the disease is clinically not uncommon, in only seven cases have morbidanatomical details been reported. Six of these reviewed by Waring, Neubuerger and Geever (1942) showed such histological variety that they felt unable to define a specific picture. Nevertheless several of the cases had shown perivascular demyelination. A very different picture was reported by Lander (1955) when he described a case of haemorrhagic encephalitis following varicella in a man of 36 years. Even in this case, however, the white matter was principally involved and there were some areas of demyelination. In the case reported below the cerebral grey matter was the site of maximal attack, illustrating yet another facet and supporting the view (Lumsden, 1958) that the essential lesion of post-exanthematous encephalitis is a vascular one.

\section{Case Report}

The patient, a girl aged 4 years and 9 months, was admitted to hospital on May 20,1954. She was a well nourished child whose only previous notable illnesses had been measles and whooping cough.

Two weeks before admission she had developed a papulo-vesicular rash on the trunk with a few lesions also on the limbs, face and scalp. At the time she had been off colour and a diagnosis of chicken pox was made. She was not kept in bed. During the second week after the appearance of the rash she had been a little unsteady, falling forwards on a number of occasions. Four days before admission she awoke with a headache, but, despite this played outside with other children. Next day she became anorexic and lethargic. Her condition deteriorated and she was admitted to hospital in a stuporose state.

On examination after admission her trunk was covered with a rash, the lesions being in various stages of healing; many of those which had resolved had left small scars. From the appearance of the rash and the mother's description of its development there was no doubt that it was a healing chickenpox exanthem. Her heart, lungs, abdomen, ears and throat were clinically normal. She was a semi-comatose child who responded only slightly to requests made during examination. Her pupils were equal and reacted to light but her eye movements were irregular and unco-ordinated. As far as examination was possible no other signs of cranial nerve lesions were detected. At this time she appeared to be able to swallow normally. The retinae were normal and the optic disc margins well defined. She had some degree of right-sided hemiparesis, the arm being more affected than the leg. Her right knee and ankle jerks were increased and both plantar responses were extensor. There was no neck stiffness but Kernig's sign was positive, more so with the left leg than with the right. A provisional diagnosis of varicella encephalitis was made and lumbar puncture performed. This yielded clear cerebrospinal fluid under increased pressure. It contained 10 lymphocytes per c.mm.; $25 \mathrm{mg}$. of protein, $70 \mathrm{mg}$. of glucose, and $700 \mathrm{mg}$. of chlorides per $100 \mathrm{ml}$. A white blood count at this time showed a total of 14,200 per c.mm. (polymorphs $88 \%$, lymphocytes $10 \%$, monocytes $2 \%$ ).

During the evening of her first day in hospital she developed convulsions, and for an hour there were periods, when she exhibited clonic movements of the right arm, leg and abdominal muscles. Her head and eyes jerked to the right; these movements occurred about once every second. At 10.20 p.m. the convulsive movements ceased spontaneously but she then appeared more deeply comatose and unable to swallow. During the night she developed further convulsions which were controlled with intramuscular phenobarbitone. To combat dehydration and maintain nutrition an intravenous drip was set up; through it $150 \mathrm{mg}$. of aureomycin were given. Her condition continued to deteriorate and further convulsions necessitated the use of intramuscular paraldehyde.

During the day of May 21 her condition remained much the same though she had no further convulsions. A further $200 \mathrm{mg}$. of aureomycin were given at 7.0 p.m., at which time she made hardly any response to external stimuli. Her pupils remained fully dilated and she appeared to have a rapidly progressive bulbar paralysis. At 5.20 a.m. the following day she died.

Necropsy. There were a few pock scars on the skin of the trunk. Apart from these and some pulmonary 
congestion, the only significant changes visible to the naked eye were in the brain. Here the grey matter of cerebral cortex and basal ganglia was pink and patchily swollen. The white matter appeared normal.

Histology. The most striking feature was the localization of the inflammatory reaction to the grey matter (Fig. 1). Here there was swelling of capillary

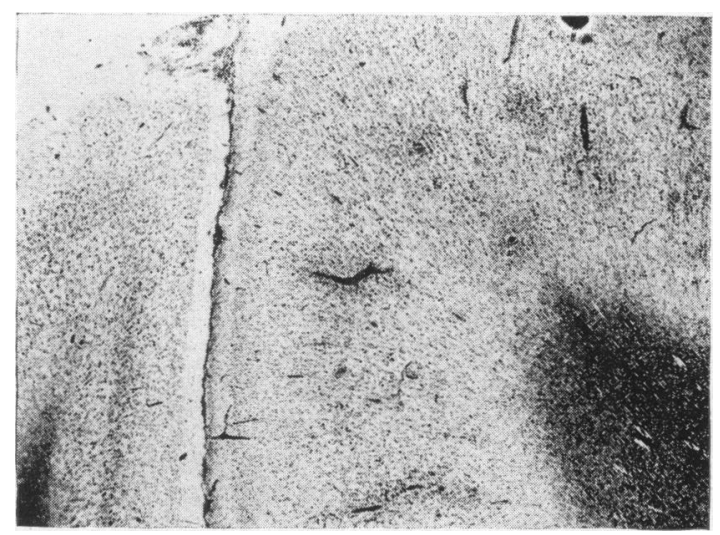

Fici. 1.-Cerebral cortex showing dilated venules and capillaries surrounded by zones of cellular reaction. The gyral white matter is free from demyelinating lesions. $\times 6$

and venular endothelium (Fig. 2). The larger vessels were surrounded by a cuff of inflammatory cells, some of which were lymphocytes and plasma cells, but most were microglia. These latter cells showed mitotic activity. In contrast, the astrocytes were not prominent having only a little more cytoplasm than normal. Within the affected areas neurones were damaged showing a loss of Nissl substance and some cytoplasmic vacuolation. There were none of the changes of specific neuronal disease and no actual destruction or phagocytosis of nerve cells.

The vascular and inflammatory lesions were most common in the deeper layers of the cortex, often ending

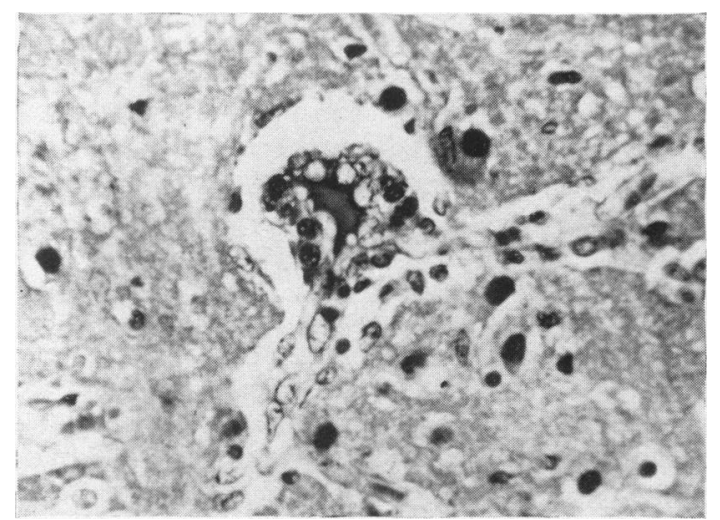

Fic. 2.-Small vessel from cortex with swollen endothelial cells and narrowed lumen $\times \mathbf{4 4 0}$.

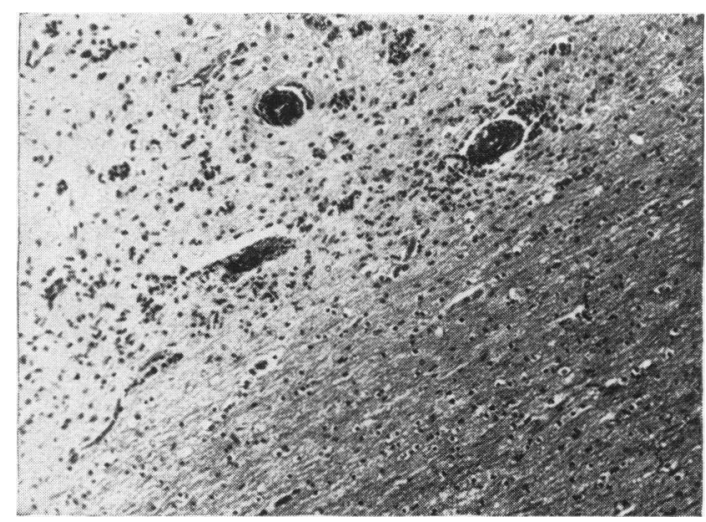

FIG. 3.-Blood vessels in deepest parts of the cortex 'cuffed' by inflammatory cells. Numerous reactive microglial cells confined to the grey matter. $\times 100$.

sharply at the edge of the white matter (Fig. 3). They were present to approximately the same degree in all lobes of both cerebral hemispheres. Over large areas the lesions were confluent and no gyrus examined was without changes. In severely affected areas there was oedema of the cortex.

Elsewhere the grey matter was less severely involved than the cerebral cortex. Vascular endothelial lesions and microglial proliferation were present in the corpus striatum, thalamus, and the head of the caudate nucleus of both hemispheres. The hypothalamic nuclei, geniculate bodies, corpora quadrigemina, corpora mamillaria, substantia nigra, and red nuclei showed only a few changes. The mid-brain, pons and medulla were also slightly affected; no lesions were found in the cerebellar cortex or nuclei.

The white matter was involved in a few places only, usually beneath severely affected cortex. Here a few vessels had swollen endothelium with a cellular infiltration of their walls and a surrounding zone of reactive microglia. Rarely there was perivascular demyelination (Fig. 4).

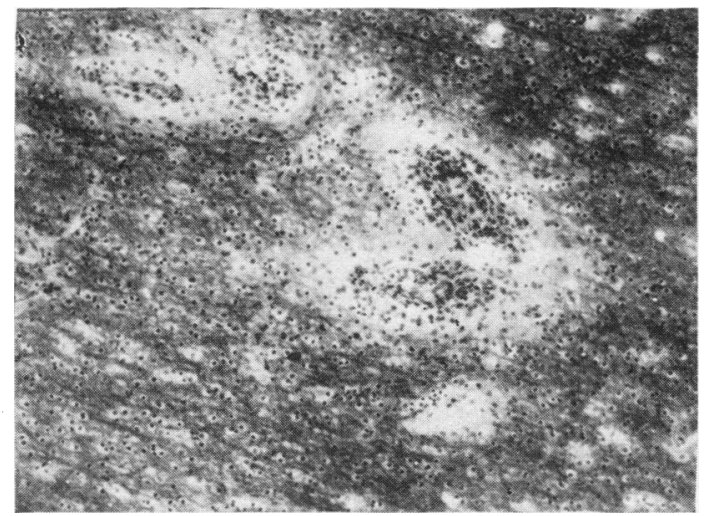

FIG. 4.-White matter of occipital lobe showing small blood vessels surrounded by lymphocytes and microglia which lie within pale perivascular zones of demyelination. Loyez $\times 100$. 
In mixed grey and white areas such as the corpus striatum the vascular lesions were more prominent in the grey matter-this predominance was greater than would be accounted for by the relative proportions of capillary bed in the two types of tissue. The cèrebellar white matter, the optic tracts and cranial nerves were normal.

The upper cervical part of the spinal cord, which was the only part available for examination, showed none of the typical vascular or glial changes and the neurones were unaffected. There was, however, a striking degeneration of the myelin sheaths of most of both dorsal and ventral spinocerebellar tracts and to a lesser extent of the posterior and lateral parts of the dorsal columns (Fig. 5). The bundles running from the upper end of

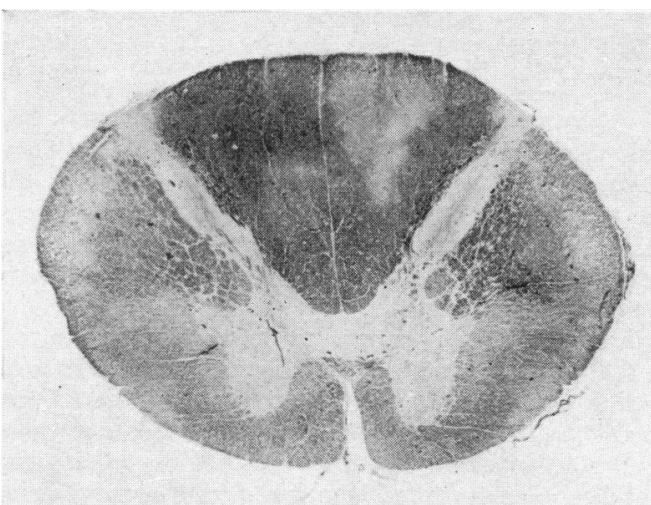

Fig: 5.-Cervical cord showing ascending tract degeneration of dorsal and lateral columns. Loyez $\times 7$.

Clarke's column to the dorsal spino-cerebellar tracts were degenerated with swollen myelin sheaths and fragmented axons. The spino-thalamic tracts were involved to a much lesser degree. Unfortunately no posterior root ganglia and no peripheral nerves were available for examination.

The meninges were infiltrated by lymphocytes, plasma cells and macrophages especially in regions where there was considerable underlying cerebral disease.

None of the viscera of the body showed any significant histological changes. A careful search did not reveal any vascular lesions comparable with those in the brain or any focal necrosis of tissue. No definite inclusion bodies could be demonstrated. An attempt to grow virus from the cerebral tissue was not successful; tissue culture terhniques were not available at the time.

\section{Discussion}

Clinically neurological involvement in varicella is most commonly of a 'cerebellar' type in which ataxia, vomiting, nystagmus and occasionally speech changes are present (Underwood, 1935). However, recovery is usual and in the few pathological reports available appropriate lesions of the cerebellum or spinal cord were not recorded. The changes in the spinal cord in this case would undoubtedly account for the ataxia which preceded the encephalitis by several days. It is unfortunate that the origins of these ascending tract degenerations could not be examined. The cerebellum itself was not primarily affected. There are three notable features of the encephalitic process; the swelling of vascular endothelium, the intense proliferation of microglia and the occasional perivenular zones of demyelination. Each of these features is present in varying degrees in the encephalitis which follows not only chickenpox but also measles and vaccination against smallpox. Appelbaum, Dolgopol and Dolgin (1949) discussing the histological changes in measles encephalitis noted that vascular changes were common and in acute cases, where death occurred early, were widely distributed irrespective of the presence or absence of demyelination. In the cases dying later in the disease perivascular demyelination was usual and often extensive. Microglial activity was always marked though only at a late stage did these cells apparently begin to function as phagocytes. This intense cellular proliferation cannot be considered a direct response to necrosis of neurones or of myelin sheaths, since in many lesions it precedes the slightest evidence of such damage.

Six earlier pathological reports of varicella encephalitis were reviewed by Appelbaum, Rachelson, and Dolgopol (1953). They observed that though perivascular demyelination was the most prominent feature, changes in the grey matter occurred in almost all.

If the encephalitis is the result of direct viral attack then some evidence should be seen in cases dying of severe generalized chickenpox. Waring et al. (1942) reported such a case; a man aged 40 years died on the eighth day of illness. There was evidence of vascular damage in organs of the body and in the brain with focal necroses and petechial haemorrhages. Capillary endothelium was swollen in the cortex and white matter and the vessels, some of which were surrounded by haemorrhage, appeared to have undergone necrosis. Elsewhere the changes in the brain were a perivascular demyelination and microglial proliferation. On the other hand two cases described by Cheatham, Weller, Dolan and Dower (1956) showed vascular endothelial damage in many organs but the brains and spinal cords were normal. In both these latter cases the damaged endothelial cells contained intranuclear viral inclusion bodies. The evidence for direct viral attack on the vessels of the central nervous system is therefore somewhat contradictory. Moreover, encephalitis may follow chickenpox when the disease has 
apparently been extremely mild as in our case or indeed may appear during the period of regression of a more severe attack. Such factors suggest that the encephalitis is the result of some secondary phenomenon probably an abnormal antigen-antibody reaction. Support for this comes from the otherwise inexplicable changes of the microglia, which, unlike other glial cells, are closely related to the reticulo-endothelial system, and may be expected to react to the same stimuli as the vascular endothelium.

This concept of a non-specific aetiology is supported by Russell (1955) who emphasized the nosological unity of acute haemorrhagic leucoencephalitis and acute disseminated encephalomyelitis of post-exanthematous type. She presented three cases in which encephalitis had followed an upper respiratory infection and in which lesions of two types were found. The one consisted of necrosis of arteriolar walls with a fibrinous, haemorrhagic and polymorphonuclear leucocytic exudation into the surrounding brain and the other a perivascular demyelination with intense microglial activity. She was able to find these two lesions characteristic of the two types of encephalitis, side by side in the same case. All her cases were adults and followed respiratory infections; none showed any evidence of an exanthematous disease. However, Lander (1955) described a case of undoubted acute haemorrhagic leuco-encephalitis of Hurst type complicating varicella. His patient was a man of 36 who developed encephalitis five days after the appearance of a typical but severe rash of varicella. Other members of his family including three children had had typical attacks. Lander described in addition to the haemorrhagic lesions, areas of perivascular demyelination; this was clearly a case of encephalitis of mixed type similar to those reported by Russell. There is, therefore, a broad group of cerebral lesions which may follow respiratory or gastro-intestinal infection in adults and the exanthematous illnesses in childhood. The interval between the onset of the primary illness and of the encephalitis varies from a few days to two or three weeks and it seems probable that the encephalitis is the result of an acquired sensitivity of the cerebral blood vessels to circulating antibody. In adults the lesion is more likely to take the form of an acute haemorrhagic leuco-encephalitis whether it follows the more common upper respiratory infection or the exanthemata as in Lander's case. In children it is most likely to be a non-haemorrhagic disseminated encephalomyelitis with perivascular demyelination as a prominent feature.

\section{Summary}

The clinical and pathological findings are reported in a case of post-varicella encephalitis in a girl of 4 years. There was little of the perivenular demyelination usually associated with post-exanthematous encephalitis, the predominant lesions being in the grey matter of cerebral cortex and basal nuclei. The conclusion reached is that the essential abnormality is damage to blood vessels possibly of an allergic nature.

We would like to thank Dr. R. I. Mackay under whose care the patient was admitted. The photomicrographs are the work of Mr. N. Mowat.

\section{REFERENCES}

Appelbaum, E., Dolgopol, V. B. and Dolgin, J. (1949). Amer. J. Dis. Child., 77, 25.

Rachelson, M. H. and Dolgopol, V. B. (1953). Amer. J. Med., 15, 223.

Cheatham, W. J., Weller, T. H., Dolan, T. F. and Dower, J. C. (1956). Amer. J. Path., 32, 1015.

Lander, H. (1955). J. Path. Bact., 70, 157

Lumsden, C. E. (1958). Proc. roy. Soc. Med., 51, 752.

Russell, D. S. (1955). Brain, 78, 369.

Underwood, E. A. (1935). Brit. J. Child. Dis., 3283.

Waring, J. I, Neubuerger, K. T. and Geever, E. F. (1942). Arch.: intern. Med., 69, 384. 